\begin{tabular}{|c|l|}
\hline Title & Conversion of Light Propagation Direction for Highly Efficient Solar Cells \\
\hline Author(s) & Suemune, Ikuo \\
\hline Citation & $\begin{array}{l}\text { A pplied Physics Express, 4(10), 102301 } \\
\text { https://doi.org/40.1143/APE.4.102301 }\end{array}$ \\
\hline Issue Date & 2011-10 \\
\hline Doc URL & http://hdl.handle.net/2115/52460 \\
\hline Rights & ○ 2011 The Japan Society of A pplied Physics \\
\hline Type & article (author version) \\
\hline File Information & APEX 2011.pdf \\
\hline
\end{tabular}

Instructions for use 


\title{
Conversion of Light Propagation Direction for Highly Efficient Solar Cells
}

\author{
Ikuo Suemune* \\ Research Institute for Electronic Science, Hokkaido University, Sapporo 001-0020, Japan
}

\begin{abstract}
Optical absorption coefficients are generally inherent to materials and are reduced for longer optical wavelengths, especially below their absorption edges. In tandem solar cells this makes thicker bottom cells necessary to improve their optical absorption efficiencies for longer optical wavelengths. This paper proposes a method to convert the propagation direction of an incident light toward the one parallel to optical absorption layers. High-efficiency optical absorption is demonstrated with thin absorption layers with this method. Drastic reduction of layer thicknesses necessary for high photoelectric conversion efficiencies will reduce the cost and improve the yield of various solar cells.

*E-mail address: isuemune@es.hokudai.ac.jp
\end{abstract}

Green technology to reduce carbon dioxide emission is now becoming more and more important and the development of highly efficient solar cells is one of the major targets in this direction. Commercial solar cells have been dominated by those based on crystalline silicon substrates. ${ }^{1)}$ However, limited silicon resources and the overall power consumption for the production of such solar cells have been controversial, and thin-film solar cells such as thin-film silicon solar cells, ${ }^{2-4)}$ dye-sensitized solar cells, ${ }^{5-7)}$ and so on have been actively studied and developed recently. The low photoelectric conversion efficiencies of these types of solar cell have been a problem, but the introduction of multijunction types such as tandem solar cells ${ }^{8,9)}$ is improving the situation.

Optical absorption is generally reduced for the lower photon energies, especially below the absorption edges of materials. For a given medium with a length $L$ and an absorption coefficient $\alpha$, the absorption ratio of an incident light is given by $1-\exp [-\alpha L]$. From this relation, we need the medium length $L$ of $\sim 2 / \alpha$ for more than $80 \%$ absorption of the incident 
light. Typical silicon thin-film tandem solar cells consist of a top cell made of $\sim 300$-nm-thick amorphous silicon (a-Si) covering the wavelength band centered at $500 \mathrm{~nm}$ and a bottom cell made of $\sim 2-\mu \mathrm{m}$-thick microcrystalline silicon ( $\mu \mathrm{c}$-Si) covering the wavelength band centered at $800 \mathrm{~nm}^{2,3)}$ The reason for the thicker bottom cell originates from the lower absorption coefficient in the longer wavelength. ${ }^{10}$ ) For example, the optical absorption coefficient of $\mu \mathrm{c}-\mathrm{Si}$ at the wavelength of $800 \mathrm{~nm}$ is $\sim 10000 \mathrm{~cm}^{-1}$. This gives $86 \%$ absorption for a thickness of $L=2 \mu \mathrm{m}$. This thicker bottom cell reduces the throughput of device fabrication and therefore increases the device cost as well as reducing the solar cell efficiencies by the recombination of photogenerated carriers during transport in the thicker layers. As a countermeasure to overcome the throughput issue, higher deposition rate techniques of $\mu \mathrm{c}-\mathrm{Si}$ have been studied, ${ }^{11-13)}$ but higher deposition rates tend to reduce photoelectric conversion efficiencies. $^{14)}$

In this paper, I propose a new method to overcome this inherent problem between the lower optical absorption coefficients and the longer optical-path lengths necessary for high-efficiency absorption. The concept is based on the conversion of an incident light propagation direction toward the one parallel to optical absorption layers with the modulation of the incident-light wave front by a periodic $\pi$-phase shift. The impact of this light propagation direction conversion layer (LPDCL) is demonstrated with finite-difference time-domain (FDTD) simulations. FDTD simulation is a computational electrodynamics modeling technique to calculate the basic Maxwell's differential equations directly with the replacement of the partial differential forms in time and space into the corresponding finite difference equations ${ }^{15,16)}$ and is known to give accurate results with small enough grid sizes.

The fundamental concept is illustrated with a simplified structure in Fig. 1. The background is assumed to have a uniform refractive index of $n_{1}$ to prevent unnecessary optical reflections and to focus on the key concept. LPDCL is introduced normal to the incident-light propagation direction and consists of periodic regions with the refractive indices of $n_{1}$ and $n_{2}$ and with the period of $D$ and the thickness of $H$. When a light wave is incident on the LPDCL and passes through the thickness $H$, the wave front experiences the lateral periodic phase shift of $2 \pi\left(n_{2}-n_{1}\right) H / \lambda$. When this phase shift is equal to $\pi$ or its odd integer multiple, the light wave in the medium just after passing through the LPDCL forms a standing wave in the direction parallel to the LPDCL. Then, this wave starts to propagate in the direction normal to the incident light propagation direction. This is the main reason to call the periodic structure the LPDCL. 


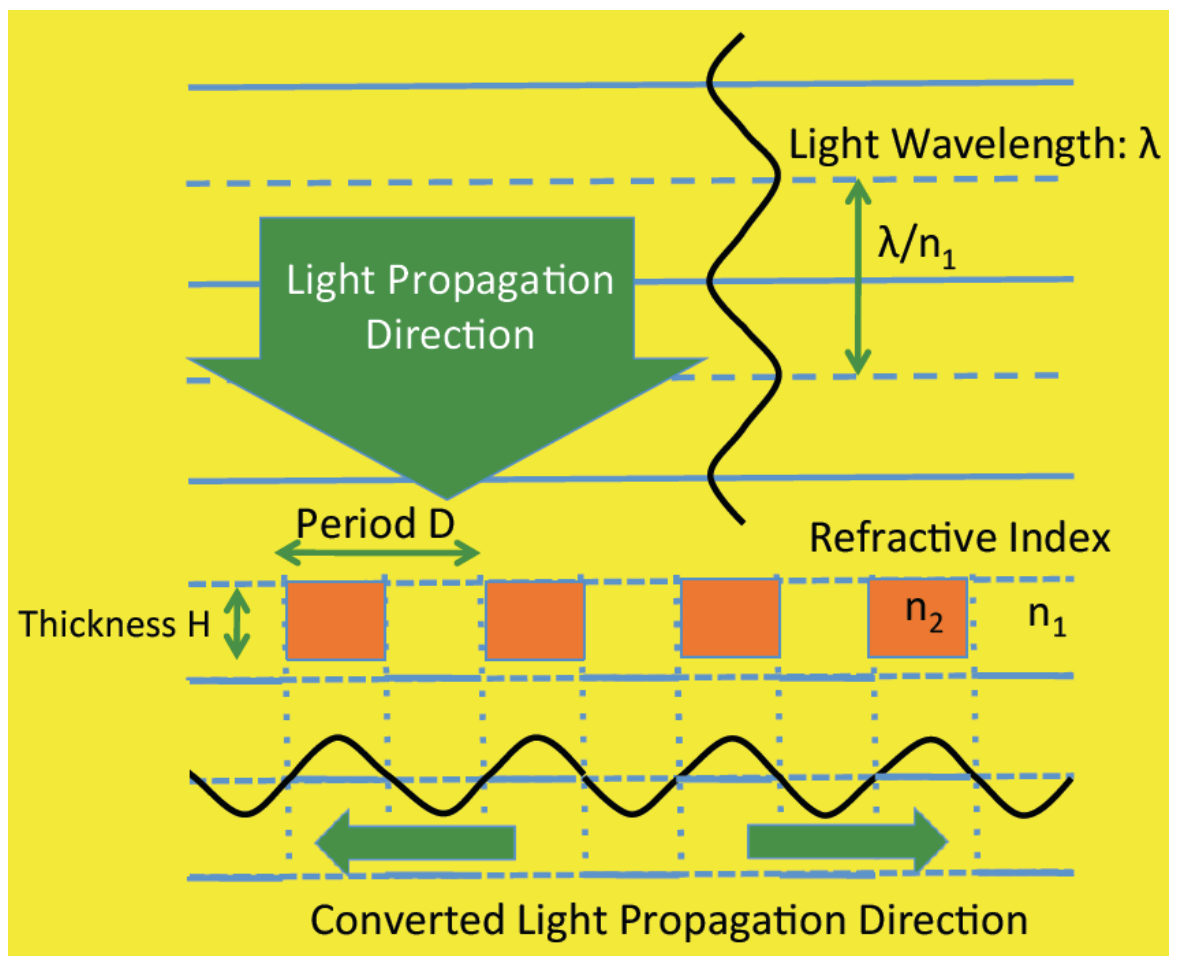

Fig. 1. Schematic of fundamental concept of light propagation direction conversion layer (LPDCL). The LPDCL consists of periodic layers with different refractive indices to convert the incident light propagation direction to the direction parallel to the conversion layer by changing the phase by $\pi$ along the LPDCL.

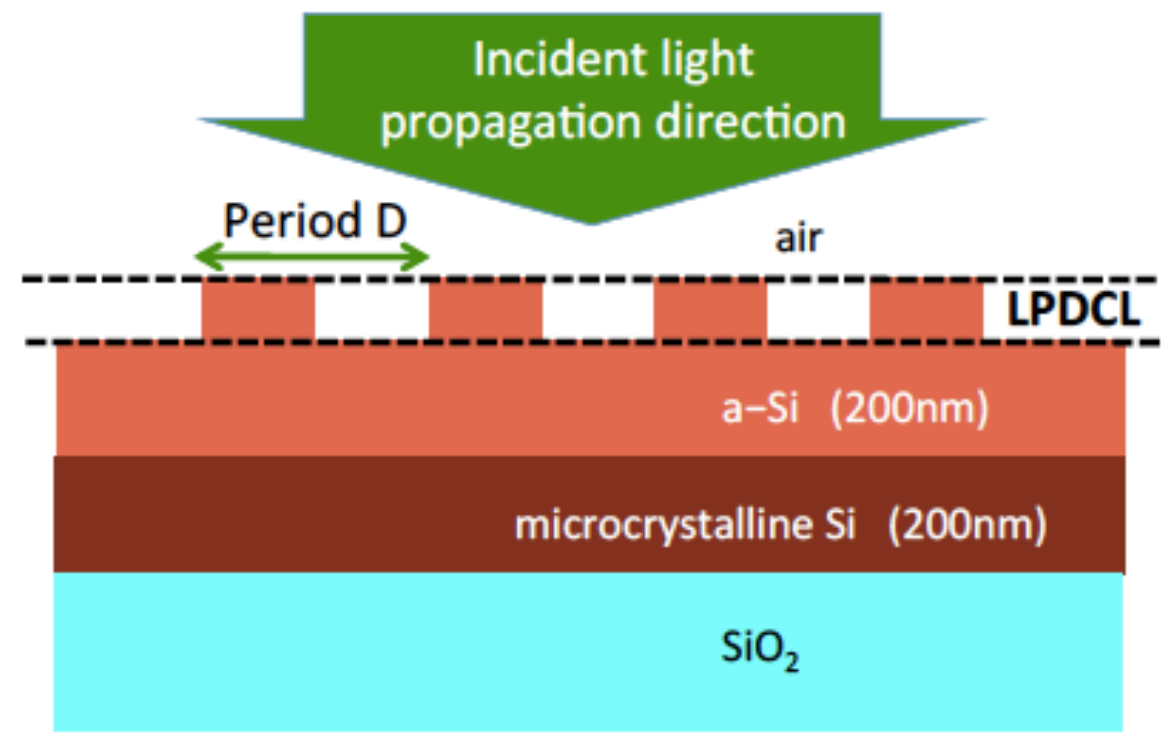

Fig. 2. Example that incorporates LPDCL. In this example, a periodic a-Si/air structure with the period D and the height $\mathrm{H}$ is assumed. Optical absorption layers are assumed to be a-Si and $\mu \mathrm{c}-\mathrm{Si}$ layers following the top-cell and bottom-cell design in usual thin-film tandem solar cells but with a much thinner bottom cell. 
Let us consider the more realistic case shown in Fig. 2. A pair of a top cell and a bottom cell formed on a glass substrate is considered but with a much thinner bottom cell, i.e., a 200-nm-thick a-Si top cell layer and a 200-nm-thick $\mu \mathrm{c}-\mathrm{Si}$ bottom cell layer. A simple LPDCL is assumed, that is, a periodic a-Si layer formed on the a-Si top cell. For the FDTD calculation at the wavelength of $800 \mathrm{~nm}$, the complex refractive index of each layer was given as follows: $4.23+0.10 \mathrm{i}$ for a-Si, $3.71+0.01 \mathrm{i}$ for $\mu \mathrm{c}-\mathrm{Si}$, and 1.45 for a glass substrate $\left(\mathrm{SiO}_{2}\right)$. The imaginary part of the refractive index is related to the optical absorption coefficient $\alpha$ by $\alpha \lambda / 4 \pi$. The refractive indices in the LPDCL are given as $n_{1}=1$ (air) and $n_{2}=4.23+0.10 \mathrm{i}$ (a-Si) in Fig. 2. Since $n_{2}$ is complex, the phase shift given by $2 \pi\left(n_{2}-n_{1}\right) H / \lambda$ is not simple but the LPDCL layer thickness $H$ was given as $125 \mathrm{~nm}$ by employing the real part of $n_{2}$, which approximately gives the $\pi$-phase difference. The grid size for the FDTD simulation was given as $1 / 60$ of $\lambda / \mathrm{n}(\mathrm{a}-\mathrm{Si})$, which is $3.2 \mathrm{~nm}$ for $\lambda=800 \mathrm{~nm}$. This was small enough to give accurate simulation results.

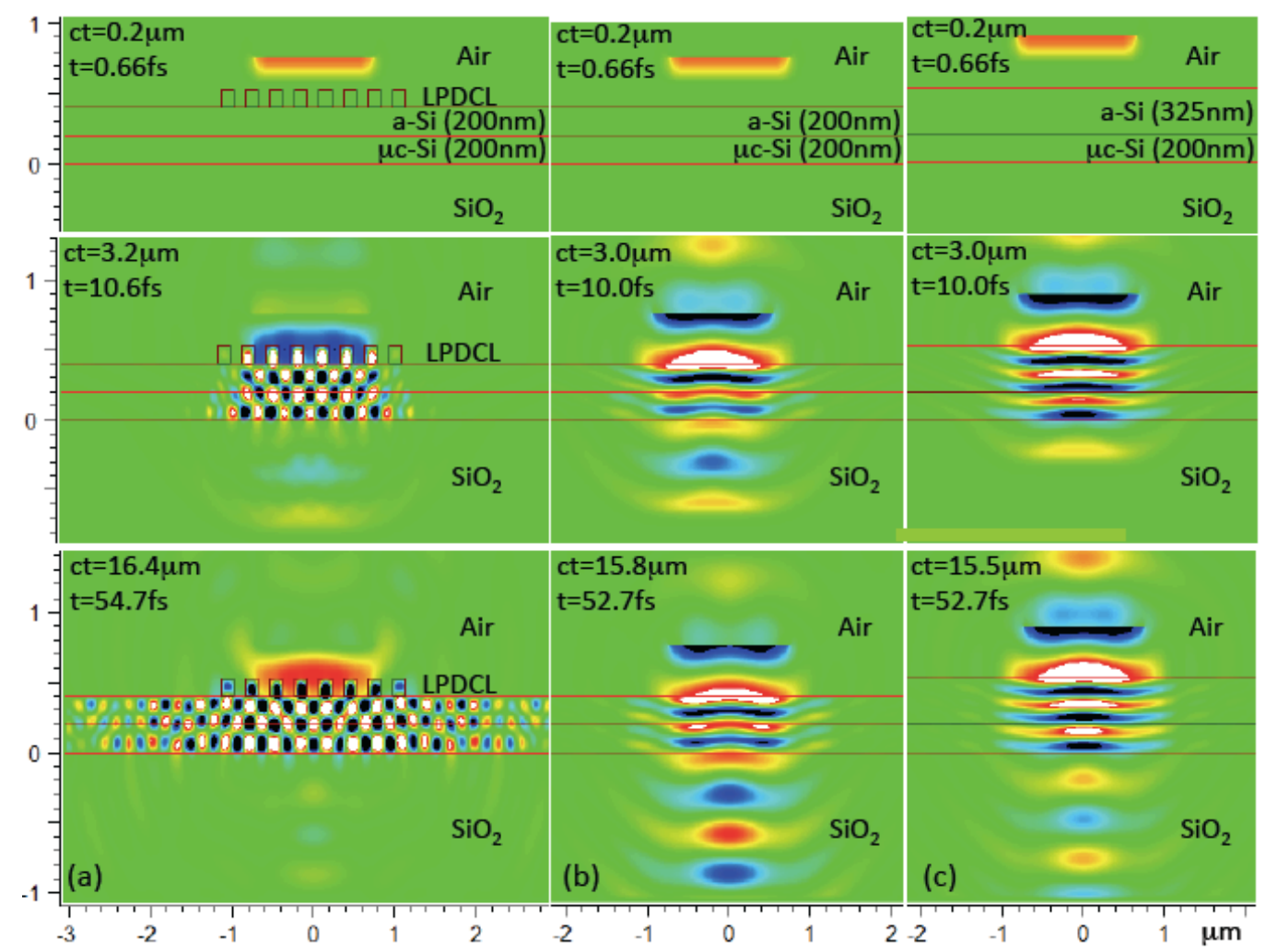

Fig. 3. FDTD simulation and demonstration of the LPDCL effect. (a) With LPDCL consists of a periodic structure of 125-nm-thick and 150-nm-wide a-Si region and 150-nm-wide air region. (b) Without LPDCL. (c) LPDCL replaced with 125-nm-thick a-Si layer. The optical absorption layers of 200-nm-thick a-Si and 200-nm-thick $\mu \mathrm{c}$-Si layers grown on a glass substrate are the same for (a), (b), and (c). 
Figure 3 shows the time-dependent downward propagation of an electromagnetic plane wave calculated with 2-dimensional modeling. Figure 3(a) is the case when the plane wave is incident on the LPDCL of $300 \mathrm{~nm}$ periodicity that consists of 150-nm-wide a-Si layers and 150-nm-wide air regions. Since incoherent light is usually incident on solar cells, finite-width plane wave incidence was considered. In this case, a light wave width covering five periods of the LPDCL was assumed and therefore eight periods of the LPDCL is enough to demonstrate its main function. The incident-light electric field parallel to the simulation plane was assumed in Fig. 3. $t$ is the light propagation time given with the unit of femtoseconds (fs) and $c t$ is the light propagation length, where $c$ is the vacuum light velocity. As the incident light propagates through the LPDCL, the wave front is modulated due to the different light velocities in the two constituent regions of the periodic structure. The phase variation of the incident light wave in the Si layers is expressed by the difference in colors. The lateral phase modulation after passing through the LPDCL is clear at $t=10.6 \mathrm{fs}$. The periodic dark and white color changes along the a-Si as well as $\mu \mathrm{c}-\mathrm{Si}$ layers correspond to the periodic lateral $\pi$-phase shift. Figure 3(a) also shows the alternate phase change with the propagation in the incidence direction. At the later time of $t=54.7 \mathrm{fs}$, the light propagation is dominated in the direction parallel to the Si layers. This simulation showed that the light transmission through the Si layers was reduced to $4.5 \%$ and the light reflection was also reduced to $6.6 \%$. The light absorption in the a-Si and $\mu \mathrm{c}-\mathrm{Si}$ layers including the a-Si regions of the LPDCL reaches as high as $88.9 \%$.

As a reference, FDTD simulations on similar structures but without the LPDCL were studied. Figure 3(b) has the same a-Si and $\mu \mathrm{c}-\mathrm{Si}$ layers as those in Fig. 3(a). In this case, the incident light was slightly diffracted laterally due to the finite beam width, but the major part was transmitted into the $\mathrm{SiO}_{2}$ glass substrate. The transmission into the glass substrate was $53.1 \%$ and was the major part, while the reflection remained at $13.5 \%$ of the incident light. Optical absorption in the a-Si and $\mu \mathrm{c}-\mathrm{Si}$ layers was limited to $33.4 \%$ due to the thin total $\mathrm{Si}$ layers. This comparison demonstrates that the phenomenon shown in Fig. 3(a) is not dominated by the optical reflection at the bottom $\mu \mathrm{c}-\mathrm{Si} / \mathrm{SiO}_{2}$ interface.

Another remaining question is the total Si-layer thickness dependence. Since the a-Si layers in the LPDCL are missing in the structure shown in Fig. 3(b), this may be the factor for the reduced light absorption in the Si layers. Therefore, the LPDCL in Fig. 3(a) was replaced with a uniform a-Si layer, as shown in Fig. 3(c), i.e., the a-Si layer thickness was increased from $200 \mathrm{~nm}$ in Fig. 3(b) to $325 \mathrm{~nm}$. The simulated overall light propagation characteristics were similar to those in Fig. 3(b), but the light reflection rather than the light transmission 
was increased at the air/a-Si interface. $48.5 \%$ of the incident light was reflected and $24.4 \%$ was transmitted into the $\mathrm{SiO}_{2}$ glass substrate. This is the reversal of the reflection and transmission properties shown in Fig. 3(b). Optical absorption in the a-Si and $\mu c-S i$ layers was reduced to $27.1 \%$ in spite of the increase in the total Si layer thickness. Therefore, the phenomenon in Fig. 3(c) is dominated by the optical reflection at the air/a-Si interface.

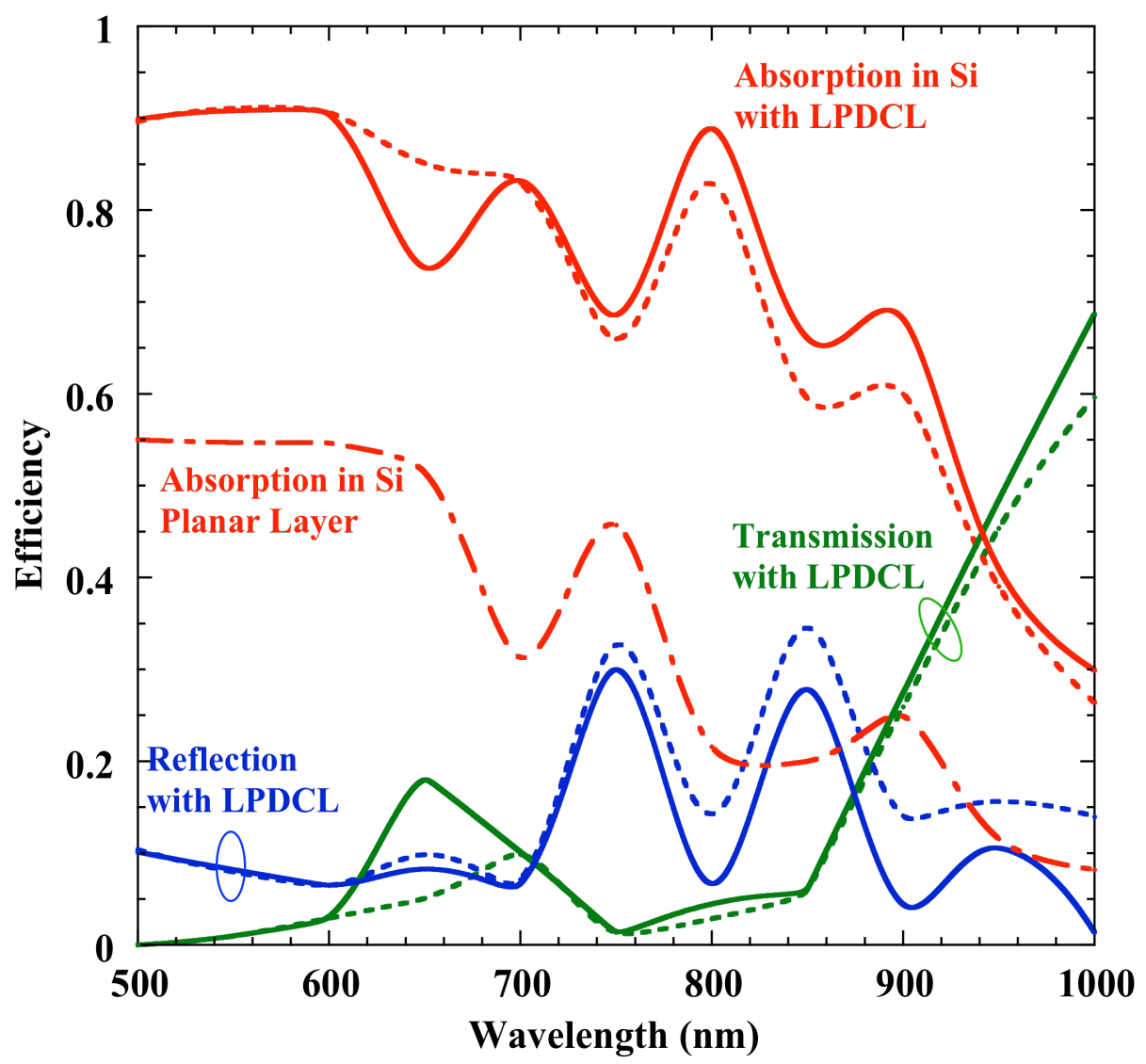

Fig. 4. Spectral dependence of optical transmission, reflection, and absorption on incident light wavelength. Structure with LPDCL for the solid lines is the same as that in Fig. 3(a) and for the dashed lines with extended periodic structure. The planar structure is that replacing the LPDCL with an additional a-Si layer with the average thickness of $63 \mathrm{~nm}$.

The spectral dependence was studied by changing the incident-light wavelength. The solid lines in Fig. 4 are the reflection, transmission, and absorption in the silicon layers calculated in the same configuration as that shown in Fig. 3(a). The dot-dash line is the absorption calculated as a reference for the Si layers without the LPDCL with the a-Si layer thickness of $263 \mathrm{~nm}$, which is the sum of the 200-nm-thick a-Si absorption layer and the average thickness of the LPDCL. Highly improved absorption efficiency is realized with the LPDCL in the wide 
bandwidth. The periodic variations are due to the internal multiple reflections within the $\mathrm{Si}$ layers. The disappearance of the periodic variation in the wavelength range shorter than 600 $\mathrm{nm}$ is due to the extremely large optical absorption coefficient more than $10^{5} \mathrm{~cm}^{-1}$ and the reduced reflectivity due to the surface periodic structure. The decreased absorption for the wavelength above $900 \mathrm{~nm}$ is due to the lower absorption coefficients, as evidenced by the increase in the transmission through the Si layers.

Here the relation of the LPDCL and grating couplers (GCs) is discussed. GCs convert guided modes to leaky modes or vise versa through gratings attached to mostly absorption-free waveguides. The main design principle of a GC is the phase matching condition along the waveguide propagating direction among a guided mode, a leaky mode, and the grating period. Gratings are generally a perturbation to guided modes and many periods more than several tens of them are necessary for the efficient mode conversion. The increase of the grating height strengthens the perturbation and reduces the necessary number of the periods, but the optimum grating height has not been discussed before. In the LPDCL the "grating" height is fixed so that the spatial periodic $\pi$-phase shift is induced in the light propagating along the silicon absorption layer and this factor is more important for the efficient mode conversion. We have studied the dependence on the number of the periodicity in the LPDCL starting from one period, and the optical absorption efficiency was saturated at $85 \sim 90 \%$ with about six periods. This is because the guided modes have very short coherence length on the order of $\mu \mathrm{m}$. This high absorption efficiency achieved with such small number of periods is possible only by setting the LPDCL height for the $\pi$-phase shift and is well fitted to solar-cell applications where incoherent light is irradiated. This situation is much different from the conventional GCs and this is the reason why GCs have not been applied to solar cells except for ref. 17 in our knowledge.

Waveguides generally support leaky modes as well as guided modes. Extension of the periodic structure to the non-irradiated surface may increase the coupling to leaky modes. The calculated results are shown by the dashed lines in Fig. 4 and compared with the solid lines without the extension. As a general trend, reflection increases and absorption decreases for wavelengths longer than $700 \mathrm{~nm}$. The important point is that the increase in reflection takes place in the irradiated area, not in the extended area (not shown). This indicates that light propagating along the silicon layers is reflected back by the outer additional periodic structures and the influence of leaky modes is negligible. This feature is also much different from conventional GCs.

The relation of the LPDCL and phase gratings (PGs) is also discussed. Moharam et. al. 
analyzed PGs with arbitrary grating cross sections and showed that an efficient conversion from the 0 -th to 1 st diffraction mode is possible by changing the grating height. ${ }^{18)}$ Catchpole also analyzed rectangular gratings with the concept of the grating modes that propagates the PG region and demonstrated the high efficiency conversion to the 1st diffraction mode by the mutual cancellation of the 0 -th and 2 nd grating modes. ${ }^{19)}$ Including these reports, the applications of diffraction gratings to solar cells discuss the high efficiency conversion to the diffraction modes with arbitrary diffraction angles under the condition that they are out of the escape cone. ${ }^{20-24)}$ However the diffracted lights are reflected back to the PGs from the counter mirrors and can be scattered out of the solar cells again. Madzharov et. al. discussed the insertion of an additional reflection grating so that such extra diffraction loss can be prevented. ${ }^{20)}$ For the normal incidence to $\mathrm{PGs}, 90^{\circ}$ diffraction of the 1 st diffraction mode may be conceivable. But it means that the diffracted light is propagating in the PG region. In this regard, the $\pi$-phase shift of the light in the light absorbing layer adjacent to but out of the LPDCL is important for realizing efficient solar cells.

The high efficiency reported in this paper also relies on the optimization method. The present scheme minimizes reflection as well as transmission and maximizes light absorption, and the simulation accuracy is maintained by examining the energy conservation of the incident Poynting vector power flow. Optimized structures are well correlated to the $\pi$-phase shift along the light absorbing layers just after passing through the LPDCL, as exemplified in Fig. 3(a). This makes it possible to realize a high light absorption efficiency of $\sim 90 \%$ with just five periods of the LPDCL.

It is clear from Fig. 3 that the function of the LPDCL does not require optical reflections from metal electrodes. This will extend the application to transparent solar cells. ${ }^{25)}$ Although the structure shown in Fig. 2 does not include p-n junctions or electrodes, the essential feature of the LPDCL is not changed with their inclusion. For example, replacement of the air regions in the LPDCL with transparent conductive oxide electrodes is possible by redesigning the LPDCL thickness. The present FDTD calculations assumed a two-dimensional structure, but the extension to LPDCLs three-dimensionally superposed in different surface directions is straightforward. This is because the main LPDCL effect is the $\pi$-phase shift of the transmitted wave front and the superposition principle of waves naturally applies. The resultant LPDCL structures may apparently resemble photonic crystals (PCs). ${ }^{24,26)}$ However the proposed LPDCL does not include any resonance effects, while the key concept of "photonic band gap" arises from resonance effects in the periodic PC structures.

There have been several unique proposals to improve solar cell photoelectric conversion 
efficiencies. Plasmonic solar cells have been actively studied recently with the capability of light trapping in ultrathin plasmonic solar cells. ${ }^{27)}$ In plasmonic devices, the presence of metal is essential. The proposal of inserting asymmetric gratings inside solar cells ${ }^{28)}$ also assumes the presence of metal. In the proposed LPDCL, the presence of metal is not essential as discussed above and therefore it can be applied in a wider range of solar cells. Since the concept of $\pi$-phase shift with an LPDCL is not dependent on the selection of materials, further improvement of the multiple-junction solar cells ${ }^{29)}$ is expected by applying LPDCL, especially to the lower photon energy band.

In summary, a new method to convert the incident-light propagation direction toward the one parallel to optical absorption layers was proposed and high-efficiency optical absorption was demonstrated with thin absorption layers with this method. This will give a breakthrough on the limiting relations between optical absorption and necessary optical lengths in various solar cells and general optical detector applications.

\section{Acknowledgments}

The author wishes to thank K. Onodera, Center for Innovation and Business Promotion, Hokkaido University, for his useful advice and suggestions and S. Ida for his assistance in the early stage of this work. 


\section{References}

1) "Advances in crystalline silicon solar cell technology for industrial mass production", NPG Asia Materials research highlight | doi:10.1038/asiamat.2010.82, Published online 22 July 2010.

2) J. Meier, S. Dubail, R. Flückiger, D. Fischer, H. Keppner, and A. Shah: Proc. 1st World Conf. Photovoltaic Energy Conversion (WCPEC-1), 1994, p. 409.

3) B. Rech, T. Repmann, M. N. van den Donker, M. Berginski, T. Kilper, J. Hüpkes, S. Calnan, H. Stiebig, and S. Wieder: Thin Solid Films 511-512 (2006) 300.

4) E. Maruyama, S. Okamoto, A. Terakawa, W. Shinohara, M. Tanaka, and S. Kiyama: Sol. Energy 74 (2002) 339.

5) M. Grätzel: J. Photochem. Photobiol. C 4 (2003) 145.

6) A. Nattestad, A. J. Mozer, M. K. R. Fischer, Y.-B. Cheng, A. Mishra, P. Bäuerle, and U. Bach: Nat. Mater. 9 (2010) 31.

7) M. A. Green, K. Emery, Y. Hishikawa, and W. Warta: Prog. Photovolt. Res. Appl. 17 (2009) 320.

8) A. S. Brown and M. A. Green: Physica E 14 (2002) 96.

9) S. P. Bremner, M. Y. Levy, and C. B. Honsberg: Prog. Photovolt. Res. Appl. 16 (2008) 225.

10) M. Ambrico, L. Schiavulli, T. Ligonzo, G. Cicala, P. Capezzuto, and G. Bruno: Thin Solid Films 383 (2001) 200.

11) C. Niikura, M. Kondo, and A. Matsuda: J. Non-Cryst. Solids 338-340 (2004) 42.

12) C. Niikura, N. Itagaki, M. Kondo, Y. Kawai, and A. Matsuda: Jpn. J. Appl. Phys. 46 (2007) 3052.

13) Y. Sobajima, S. Nakano, M. Nishio, Y. Tanaka, T. Toyama, and H. Okamoto: J.

Non-Cryst. Solids 354 (2008) 2407.

14) Y. Hishikawa, M. Sasaki, S. Tsuge, S. Okamoto, and S. Tsuda: Mater. Res. Soc. Symp.

Proc. 297 (1993) 779.

15) K. Yee: IEEE Trans. Antennas Propag. 14 (1966) 302.

16) A. Taflove: IEEE Trans. Electromagnetic Compatibility 22 (1980) 191.

17) S. H. Lim, D. Derkacs, and E. T. Yu: J. Appl. Phys. 105 (2009) 073101.

18) M. G. Moharam and T. K. Gaylord: J. Opt. Soc. Am. 72 (1982) 1385.

19) K. R. Catchpole: J. Appl. Phys. 102 (2007) 013102.

20) D. Madzharov, R. Dewan, and D. Knipp: Opt. Express 19 (2011) A95.

21) C. Eisele, C. E. Nebel, and M. Stutzmann: J. Appl. Phys. 89 (2001) 7722. 
22) C. Haase and H. Stiebig: Prog. Photovolt. Res. Appl. 14 (2006) 629.

23) L. Zeng, Y. Yi, C. Hong, J. Liu, N. Feng, X. Duan, L. C. Kimerling, and B. A. Alamariu: Appl. Phys. Lett. 89 (2006) 111111.

24) E. Yablonovitch: Phys. Rev. Lett. 58 (1987) 2059.

25) Fraunhofer-Gesellschaft (2009, June 16). Transparent Solar Cells Made For Windows.

ScienceDaily. Retrieved March 20, 2011, from http://www.sciencedaily.com/releases/2009/06/090610161004.htm

26) S. John: Phys. Rev. Lett. 58 (1987) 2486.

27) V. E. Ferry, M. A. Verschuuren, H. B. T. Li, E. Verhagen, R. J. Walters, R. E. I.

Schropp, H. A. Atwater, and A. Polman: Opt. Exp. 18 (2010) A237.

28) T. Söderström, F.-J. Haug, X. Niquille, V. Terrazzoni, and C. Ballif: Appl. Phys. Lett. 94 (2009) 063501.

29) T. Takamoto, T. Agui, A. Yoshida, K. Nakaido, H. Juso, K. Sasaki, K. Nakamora, H. Yamaguchi, T. Kodama, H. Washio, M. Imaizumi, and M.

Takahashi: Proc. 35 ${ }^{\text {th }}$ IEEE Photovoltaic Specialists Conference (PVSC), 2010, p. 412. 